\title{
Message From the Editor-in-Chief
}

Editorial Statistics and Best Reviewers Award for 2019

Toyoaki Murohara

\section{Reviews}

The Asia-Pacific Society of Cardiology (APSC) Expert Committee Consensus Recommendations for Assessment of Suspected Acute Coronary Syndrome Using High-Sensitivity Cardiac Troponin T in the Emergency Department

Wei Chieh Jack Tan, Kenji Inoue, Laila Abde/Wareth, Evangelos Giannitsis, Sazzli Kasim, Masayuki Shiozaki, Tar Choon Aw, Federick Cheng, Ho Thuong Dung, Yi-Heng Li, Swee Han Lim, Antonia Anna Lukito, Martin Than, Fang-Yeh Chu, Tom Devasia, Chien-Chang Lee, Arintaya Phrommintikul, Jong-Chan Youn, Derek $P$. Chew

Strengths and Opportunities of Network Medicine in Cardiovascular Diseases

Giuditta Benincasa, Raffaele Marfella, Nunzia Della Mura, Concetta Schiano, Claudio Napoli

\section{Editorials}

Individualized Duration of Dual Antiplatelet Therapy Guided by Risk Scores - Ready for Prime Time? -

Sergio Buccheri, Stefan James

Local Epicardial Adipose Tissue Deposits and Left Ventricular Diastolic Function in Patients With Preserved Left Ventricular Ejection Fraction

Toshiaki Nakajima, Shigeru Toyoda, Teruo Inoue

Should Patients Choose Lung Transplantation or Optimal Drug Therapy? - The Clinical Situation in the Treatment of Pulmonary Arterial Hypertension in Japan -

Hisataka Maki

\section{Original Articles}

\section{Cardiovascular Intervention}

Efficacy and Safety of Guideline-Recommended Risk Score-Directed Dual Antiplatelet Therapy After 2nd-Generation Drug-Eluting Stents

Ji-Yong Jang, Byoung-Kwon Lee, Jung-Sun Kim, Dong-Ho Shin, Sung-Jin Hong, Chul-Min Ahn, Byeong-Keuk Kim, Young-Guk Ko, Donghoon Choi, Myeong-Ki Hong, Kyung Woo Park, Hyeon-Cheol Gwon, Hyo-Soo Kim, Hyuck Moon Kwon, Yangsoo Jang

Comparison of Clinical Characteristics of Stent Thrombosis Between the Right Coronary Artery and the

Left Coronary Artery - A Subanalysis of the REAL-ST Registry -

Yusuke Watanabe, Kenichi Sakakura, Hideo Fujita, Masanobu Ohya, Kazunori Horie, Futoshi Yamanaka, Gaku Nakazawa, Hiromasa Otake, Hiroki Shiomi, Masahiro Natsuaki, Kenji Ando, Kazushige Kadota, Shigeru Saito, Takeshi Kimura, Shoichi Kuramitsu on behalf of the REAL-ST Registry Investigators

Ventricular Fibrillation During Optical Coherence Tomography/Optical Frequency Domain Imaging - A Large Single-Center Experience -

Noriko Terada, Tatsuhiko Kuramochi, Tomoyo Sugiyama, Yoshihisa Kanaji, Masahiro Hoshino, Eisuke Usui, Masao Yamaguchi, Masahiro Hada, Toru Misawa, Yohei Sumino, Hidenori Hirano, Kai Nogami, Hiroki Ueno, Nobutaka Wakasa, Masahiro Hosokawa, Tadashi Murai, Tetsumin Lee, Taishi Yonetsu, Kazuhiko Kobashi, Tsunekazu Kakuta 


\section{Cardiac Rehabilitation}

Pulsed Electromagnetic Fields Increase Angiogenesis and Improve Cardiac Function After Myocardial Ischemia in Mice

Lihong Peng, Chenying Fu, Zejun Liang, Qing Zhang, Feng Xiong, Li Chen, Chengqi He, Quan Wei

\section{Heart Failure}

Incidence and Clinical Significance of 30-Day and 90-Day Rehospitalization for Heart Failure Among Patients With Acute Decompensated Heart Failure in Japan — From the NARA-HF Study -

Satomi Ishihara, Rika Kawakami, Maki Nogi, Kaeko Hirai, Yukihiro Hashimoto, Yasuki Nakada, Hitoshi Nakagawa, Tomoya Ueda, Taku Nishida, Kenji Onoue, Tsunenari Soeda, Satoshi Okayama, Makoto Watanabe, Yoshihiko Saito

Association of Local Epicardial Adipose Tissue Depots and Left Ventricular Diastolic Performance in Patients With Preserved Left Ventricular Ejection Fraction

Gulinu Maimaituxun, Hirotsugu Yamada, Daiju Fukuda, Shusuke Yagi, Kenya Kusunose, Yukina Hirata, Susumu Nishio, Takeshi Soeki, Hiroaki Masuzaki, Masataka Sata, Michio Shimabukuro

\section{Metabolic Disorder}

Midkine Inhibits Cholesterol Efflux by Decreasing ATP-Binding Membrane Cassette Transport Protein A1 via Adenosine Monophosphate-Activated Protein Kinase/Mammalian Target of Rapamycin Signaling in Macrophages

Han-xiao Ou, Qin Huang, Chu-hao Liu, Ji Xiao, Yun-cheng Lv, Xuan Li, Li-Ping Lei, Zhong-cheng Mo

\section{Myocardial Disease}

Co-Phenotype of Left Ventricular Non-Compaction Cardiomyopathy and Atypical Catecholaminergic Polymorphic Ventricular Tachycardia in Association With R169Q, a Ryanodine Receptor Type 2 Missense Mutation

Yoshihiro Nozaki, Yoshiaki Kato, Kiyoshi Uike, Kenichiro Yamamura, Masahiro Kikuchi, Maki Yasuda, Seiko Ohno, Minoru Horie, Takashi Murayama, Nagomi Kurebayashi, Hitoshi Horigome

\section{Pediatric Cardiology and Adult Congenital Heart Disease}

Simultaneous Volumetric and Functional Assessment of the Right Ventricle in Hypoplastic Left Heart Syndrome After Fontan Palliation, Utilizing 3-Dimensional Speckle-Tracking Echocardiography

Tomoyuki Sato, Renzo JC Calderon, Berthold Klas, Gianni Pedrizzetti, Anirban Banerjee

\section{Pulmonary Circulation}

Marked Reduction of Pulmonary Artery Pressure After Registration for Lung Transplantation Is Associated With Long-Term Survival in Patients With Pulmonary Arterial Hypertension - Cohort Study -

Satoshi Akagi, Hiromi Matsubara, Kazufumi Nakamura, Takahiro Oto, Kentaro Ejiri, Hiroshi Ito

\section{Valvular Heart Disease}

Age-Related Differences in the Effects of Initial Aortic Valve Replacement vs. Conservative Strategy on Long-Term Outcomes in Asymptomatic Patients With Severe Aortic Stenosis

Akihiro Kushiyama, Tomohiko Taniguchi, Takeshi Morimoto, Hiroki Shiomi, Kenji Ando, Norio Kanamori, Koichiro Murata, Takeshi Kitai, Yuichi Kawase, Chisato Izumi, Makoto Miyake, Hirokazu Mitsuoka, Masashi Kato, Yutaka Hirano, Shintaro Matsuda, Tsukasa Inada, Kazuya Nagao, Hiroshi Mabuchi, Yasuyo Takeuchi, Keiichiro Yamane, Mamoru Toyofuku, Mitsuru Ishii, Eri Minamino-Muta, Takao Kato, Moriaki Inoko, Tomoyuki Ikeda, Akihiro Komasa, Katsuhisa Ishii, Kozo Hotta, Nobuya Higashitani, Yoshihiro Kato, Yasutaka Inuzuka, Toshikazu Jinnai, Yuko Morikami, Naritatsu Saito, Kenji Minatoya, Takeshi Kimura on behalf of the CURRENT AS Registry Investigators

Incidence, Risk Factors and Subsequent Prognostic Impact of New-Onset Atrial Fibrillation in Infective Endocarditis Xue-biao Wei, Jie-leng Huang, Yuan-hui Liu, Chong-yang Duan, Ze-dazhong Su, Yu Wang, Dan-qing Yu, Ji-yan Chen 
Importance of Preoperative Computed Tomography Assessment of the Membranous Septal Anatomy in Patients Undergoing Transcatheter Aortic Valve Replacement With a Balloon-Expandable Valve Tomonori Miki, Keitaro Senoo, Takashi Ohkura, Yoshito Kadoya, Nobuyasu Ito, Kensuke Kuwabara, Naohiko Nakanishi, Kan Zen, Takeshi Nakamura, Tetsuhiro Yamano, Hirokazu Shiraishi, Takeshi Shirayama, Satoaki Matoba

Contemporary Outcomes of Surgical Aortic Valve Replacement in Japan

Yoshiyuki Tokuda, Hiroyuki Yamamoto, Hiroaki Miyata, Akihiko Usui, Noboru Motomura, The Japan Cardiovascular Surgery Database Organization

\section{Vascular Biology and Vascular Medicine}

Changing Treatment Patterns in Patients With Venous Thromboembolism in Taiwan Cheng-Han Lee, Ching-Chang Fang, Liang-Miin Tsai, Hui-Wen Lin, Po-Sheng Chen, Sheng-Hsiang Lin, Yi-Heng Li

\section{Images in Cardiovascular Medicine}

Hitting Two Birds With One Stone - Pre-Existing Paravalvular Leak Closed by Valve-in-Valve Implantation -

Abdisamad A. Osoble, Arun Kumar, Thomas Bartel

Cardiac Rupture Due to Side Branch Occlusion After Stent Implantation - The Crime of Jailed Stent Kumiko Asakura, Taku Homma, Naotaka Akutsu, Daisuke Fukamachi, Shunsuke Ozaki, Hiroshi Ohta, Yasuo Okumura, Hiroyuki Hao

Keloid as an Underrecognized Potential Risk Factor for Post-Procedural Pulmonary Vein Stenosis Hiro Yamasaki, Tomoya Hoshi, Kazutaka Aonuma, Akihiko Nogami, Masaki leda

Acute Popliteal Artery Thrombosis After Arthroscopic Anterior Cruciate Ligament Reconstruction Kanichi Otowa, Yoshinobu Maruhashi, Akio Chikata, Michiro Maruyama, Kazuo Usuda

Extremely Rare Case of Submitral Aneurysm With Left Atrium Communication in a Japanese Patient Yutaka Matsuhiro, Ryu Shutta, Kyosuke Yanagawa, Hitoshi Nakamura, Koji Yasumoto, Masaki Tsuda, Akihiro Tanaka, Naotaka Okamoto, Yasuharu Matsunaga-Lee, Masamichi Yano, Masaki Yamato, Yasuyuki Egami, Masami Nishino, Jun Tanouchi

\section{JCS Guidelines}

JCS 2017 Guideline on Management of Vasculitis Syndrome — Digest Version -

Mitsuaki Isobe, Koichi Amano, Yoshihiro Arimura, Akihiro Ishizu, Shuichi Ito, Shinya Kaname, Shigeto Kobayashi, Yoshinori Komagata, Issei Komuro, Kimihiro Komori, Kei Takahashi, Kazuo Tanemoto, Hitoshi Hasegawa, Masayoshi Harigai, Shouichi Fujimoto, Tatsuhiko Miyazaki, Tetsuro Miyata, Hidehiro Yamada, Akitoshi Yoshida, Takashi Wada, Yoshinori Inoue, Haruhito A. Uchida, Hideki Ota, Takahiro Okazaki, Mitsuho Onimaru, Tamihiro Kawakami, Reiko Kinouchi, Atsushi Kurata, Hisanori Kosuge, Ken-ei Sada, Kunihiro Shigematsu, Eiichi Suematsu, Eijun Sueyoshi, Takahiko Sugihara, Hitoshi Sugiyama, Mitsuhiro Takeno, Naoto Tamura, Michi Tsutsumino, Hiroaki Dobashi, Yoshikazu Nakaoka, Kenji Nagasaka, Yasuhiro Maejima, Hajime Yoshifuji, Yoshiko Watanabe, Shoichi Ozaki, Takeshi Kimura, Hiroshi Shigematsu, Keiko Yamauchi-Takihara, Toyoaki Murohara, Shin-ichi Momomuraon behalf of the JCS Joint Working Group

\section{Corrigendum}

